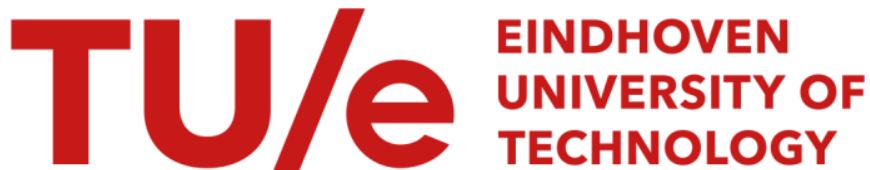

\section{Ultrafast magneto-optics in nickel : magnetism or optics?}

\section{Citation for published version (APA):}

Koopmans, B., Kampen, van, M., Kohlhepp, J. T., \& Jonge, de, W. J. M. (2000). Ultrafast magneto-optics in nickel : magnetism or optics? Physical Review Letters, 85(4), 844-847.

https://doi.org/10.1103/PhysRevLett.85.844

DOI:

10.1103/PhysRevLett.85.844

Document status and date:

Published: 01/01/2000

\section{Document Version:}

Publisher's PDF, also known as Version of Record (includes final page, issue and volume numbers)

\section{Please check the document version of this publication:}

- A submitted manuscript is the version of the article upon submission and before peer-review. There can be important differences between the submitted version and the official published version of record. People interested in the research are advised to contact the author for the final version of the publication, or visit the $\mathrm{DOI}$ to the publisher's website.

- The final author version and the galley proof are versions of the publication after peer review.

- The final published version features the final layout of the paper including the volume, issue and page numbers.

Link to publication

\section{General rights}

Copyright and moral rights for the publications made accessible in the public portal are retained by the authors and/or other copyright owners and it is a condition of accessing publications that users recognise and abide by the legal requirements associated with these rights.

- Users may download and print one copy of any publication from the public portal for the purpose of private study or research.

- You may not further distribute the material or use it for any profit-making activity or commercial gain

- You may freely distribute the URL identifying the publication in the public portal.

If the publication is distributed under the terms of Article 25fa of the Dutch Copyright Act, indicated by the "Taverne" license above, please follow below link for the End User Agreement:

www.tue.nl/taverne

Take down policy

If you believe that this document breaches copyright please contact us at:

openaccess@tue.nl

providing details and we will investigate your claim. 


\title{
Ultrafast Magneto-Optics in Nickel: Magnetism or Optics?
}

\author{
B. Koopmans,* M. van Kampen, J. T. Kohlhepp, and W. J. M. de Jonge \\ Eindhoven University of Technology, Department of Applied Physics, COBRA Research Institute, \\ P.O. Box 513, $5600 \mathrm{MB}$, Eindhoven, The Netherlands
}

(Received 22 February 2000)

\begin{abstract}
Several magnetic and optical processes contribute to the magneto-optical response of nickel thin films after excitation by a femtosecond laser pulse. We achieved a first complete identification by explicitly measuring the time-resolved Kerr ellipticity and rotation, as well as its temperature and magnetic field dependence in epitaxially grown (111) and $(001)$ oriented $\mathrm{Cu} / \mathrm{Ni} / \mathrm{Cu}$ wedges. The first hundreds of femtoseconds the response is dominated by state filling effects. The true demagnetization takes approximately $0.5-1 \mathrm{ps}$. At the longer (sub-ns) time scales the spins are found to precess in their anisotropy field. Simple and transparent models are introduced to substantiate our interpretation.
\end{abstract}

PACS numbers: 75.40.Gb, 75.70. $-\mathrm{i}, 76.50 .+\mathrm{g}, 78.47 .+\mathrm{p}$

The dynamics of itinerant ferromagnets down to femtosecond time scales is one of the challenging issues of today's magnetism, with potential impact on the future of magnetic recording and spin electronics. Several groups have shown that the magneto-optical (MO) contrast of nickel is reduced within tens to hundreds of femtoseconds after excitation by an intense laser pulse [1-4]. In most papers to date a direct relation between the MO contrast and the magnetization $(\vec{M})$ is assumed. This would mean that the magnetization relaxation $\left(\tau_{M}\right)$ proceeds much faster than the electron-phonon equilibration $\left(\tau_{\mathrm{ep}} \sim 1 \mathrm{ps}\right)$, and thus cannot be accounted for by spin-lattice relaxation $\left(\tau_{\mathrm{sl}}\right)$. Therefore, purely electronic mechanisms have been suggested, such as a direct electron-spin scattering channel [1], Stoner excitations [5], and an effective spin "temperature" being governed entirely by the electron temperature [3]. We believe, however, that a purely electronic mechanism is rather unlikely in its naive form, because the total angular momentum of the electron system $\vec{J}_{e}$ has to be conserved if interactions with the lattice or any other external degree of freedom are neglected.

The recent debate about conflicting interpretations motivated us to perform more detailed time-resolved MO Kerr effect (TRMOKE) experiments, with the aim to identify the various contributions to the MO dynamics. In this Letter, we demonstrate that differences between the induced MO rotation and ellipticity can be used as a unique fingerprint of "nonmagnetic" contributions, not directly scaling with the true demagnetization. Furthermore, we find that a simple optical "heating" pulse can trigger a rich spectrum of spin-dependent processes. We specifically exploited epitaxial (111) and (001) $\mathrm{Cu} / \mathrm{Ni} / \mathrm{Cu}$ wedges, in order to study interface-related spin scattering, the role of thermal diffusion, and the influence of magnetic anisotropy on the spin dynamics, by performing TRMOKE as a function of the Ni thickness, $d_{\mathrm{Ni}}$. Of particular interest here is the twofold reorientation transition in this system. $\vec{M}$ rotates from in plane, to perpendicular, and via a canted state back to in plane while increasing $d_{\mathrm{Ni}}$, due to an interplay between the various contributions to the uniaxial anisotropy (shape, interface, and strain related) and the cubic crystalline anisotropy [6].

By means of molecular beam epitaxy Ni wedges with a thickness ranging from 0 to $15 \mathrm{~nm}$ were grown on $\mathrm{Cu}(111)$ and (001), and capped by $3 \mathrm{~nm}$ of $\mathrm{Cu}$. Epitaxial growth was checked in situ by LEED. The equilibrium magnetic properties were verified ex situ by standard MOKE. The (001) samples show an abrupt change from a perpendicular (with square hysteresis loop) to a canted orientation at $d_{\mathrm{Ni}} \approx 8 \mathrm{~nm}$, whereas for (111) a pure single domain perpendicular orientation is not observed, and a more vague transition occurs at $d_{\mathrm{Ni}} \approx 5 \mathrm{~nm}$ (see also Fig. 3). TRMOKE experiments were performed with a 70 femtosecond Ti:sapphire laser system $(82 \mathrm{MHz}, \hbar \omega \sim$ $1.7 \mathrm{eV}$ ) in the "double-modulation" configuration as described in [4]. In this Letter we report on experiments in a polar geometry, in which a high aperture laser objective $[\mathrm{NA}=0.38$ ] is mounted inside the hollow pole tip of an electromagnet (Fig. 1, inset). The pump and

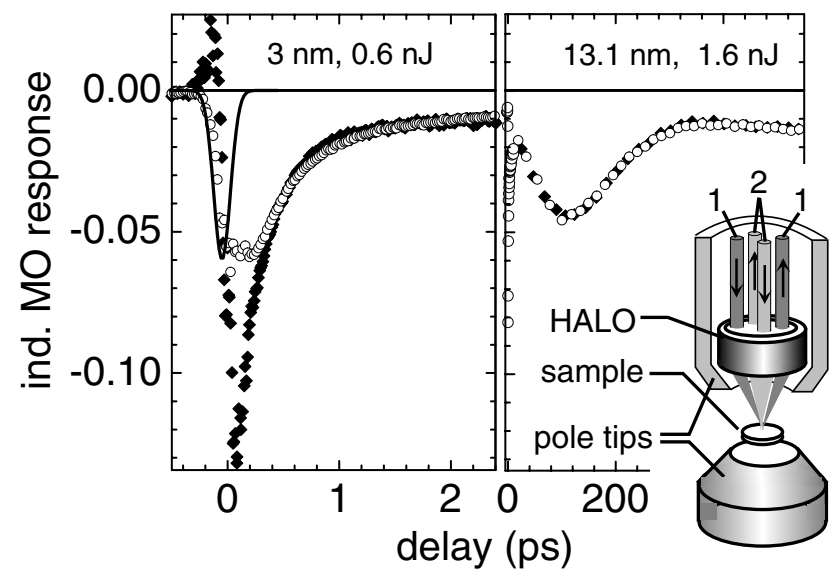

FIG. 1. Comparison of the induced ellipticity $\left(\Delta \psi^{\prime \prime} / \psi_{0}^{\prime \prime}\right.$, open circles) and rotation $\left(\Delta \psi^{\prime} / \psi_{0}^{\prime}\right.$, filled diamonds) as a function of pump-probe delay time, for a (111) oriented film at the thicknesses and pulse energies indicated. The thick line represents the pump-probe cross correlation trace. The inset depicts the experimental configuration with pump ("1") and probe (" 2 ") beams. 
probe pulses (intensity ratio $>20: 1$ ) are focused down to overlapping spots of a radius $r \approx 5 \mu \mathrm{m}$. We verified that the induced MO response scaled linearly with the pump fluence applied $\left(\mathcal{F} \leq 1.5 \mathrm{~mJ} / \mathrm{cm}^{2}\right)$. The pump beam is mechanically chopped at $60 \mathrm{~Hz}$, while the polarization of the probe is modulated at $50 \mathrm{kHz}$ by a photoelastic modulator (PEM). We define a complex polarization rotation $\psi=\psi^{\prime}+i \psi^{\prime \prime}$, and distinguish between static and pumpinduced contributions according to $\psi=\psi_{0}+\Delta \psi$. The rotation $\left(\psi^{\prime}\right)$ and ellipticity $\left(\psi^{\prime \prime}\right)$ are obtained from the signals at 50 and $100 \mathrm{kHz}$, respectively, which are measured by means of a Si photodiode and two lock-in amplifiers in series. The first lock-in amplifier, referenced by the PEM, is used to measure $\psi_{0}$. Its analog output is fed to the second lock-in amplifier (referenced by the chopper) which displays $\Delta \psi[4]$.

A typical result of the time-resolved $\mathrm{MO}$ response is presented in Fig. 1. At zero time delay between pump and probe $(t=0)$ an instantaneous decrease of $\Delta \psi^{\prime \prime}$ is found, followed by a rapid recovery of the signal. This behavior is in agreement with other recent work [1-3]. However, for films with a canted spin orientation we find that beyond 30 ps the signal starts to rise again and finally dies out in a (heavily) damped oscillatory fashion.

An explicit comparison between $\Delta \psi^{\prime}$ and $\Delta \psi^{\prime \prime}$, such as displayed in Fig. 1, is extremely useful in resolving the underlying mechanisms. We note that if a direct relation between the MO response and $\vec{M}$ were to hold, then this would be valid both for $\psi^{\prime}$ and $\psi^{\prime \prime}$. As a consequence, the equality $\Delta \psi^{\prime} / \psi_{0}^{\prime}=\Delta \psi^{\prime \prime} / \psi_{0}^{\prime \prime}$ would have to be fulfilled independent of $t$. Figure 1 shows that the equality exactly holds down to a few picoseconds. In sharp contrast to this, a completely different profile is observed during the first hundreds of femtoseconds, while small (though reproducible) differences are extended up to $\sim 1.5 \mathrm{ps}$. This demonstrates that-in contrast with previous reportsthe loss of MO contrast cannot directly be related to an instantaneous demagnetization. In order to facilitate a convenient presentation we propose the following conjecture, which will be confirmed below. At $t<0.5 \mathrm{ps}$, the high excitation density results in a spin-resolved depletion of the oscillator strength, a phenomenon we denote as "dichroic bleaching." At the intermediate $(>1 \mathrm{ps})$ and long time scale $(>50 \mathrm{ps})$ the effects are ascribed to truly magnetic effects, viz., demagnetization and spin precession, respectively.

We first concentrate on the intermediate time scale $(0.5-10 \mathrm{ps})$. In this range, the magnitude of $\Delta \psi^{\prime \prime} / \psi_{0}^{\prime \prime}$, as well as its temperature dependence will be shown to be compatible with that of thermally induced demagnetization. Although we realize the limited validity of equilibrium concepts like temperature for the initial electron and phonon populations that may be far from thermal equilibrium, a description of the "magnetization" as a function of temperature may become meaningful for $t>\tau_{\mathrm{ep}} \approx 0.5 \mathrm{ps}$. Assuming a linear relation between
$\Delta \psi^{\prime \prime}$, the induced magnetization $(\Delta M)$ and heating $(\Delta T)$, i.e., in the small-fluence limit, one obtains

$$
\frac{\Delta \psi^{\prime \prime}}{\psi_{0}^{\prime \prime}} \approx \frac{\Delta M}{M} \approx \frac{d M(T) / d T}{M} \Delta T .
$$

The $T$ dependence observed, e.g., in Fig. 2(c) agrees relatively well with this simple model. Experimentally we find, for $t=2-5 \mathrm{ps} \Delta \psi^{\prime \prime}(370 \mathrm{~K}) / \Delta \psi^{\prime \prime}(300 \mathrm{~K}) \approx$ $1.5 \pm 0.1$, whereas substitution of the equilibrium $M(T)$ from Refs. [7,8] in Eq. (1) yields $1.55 \pm 0.10$. This agreement serves as a first indication that a real demagnetization occurs within a few ps.

Figure 2(e) shows a slower decay of $\left|\Delta \psi^{\prime \prime} / \psi_{0}^{\prime \prime}\right|$ at increasing $d_{\mathrm{Ni}}$. More specifically, Fig. 3 demonstrates that $\Delta \psi^{\prime \prime} / \psi_{0}^{\prime \prime}$ at 5 ps scales with the fraction of the incident light absorbed in the Ni thin film $\left(f_{\text {abs }}\right.$, as calculated with an optical transfer-matrix approach, and literature values for the respective dielectric constants). The observation

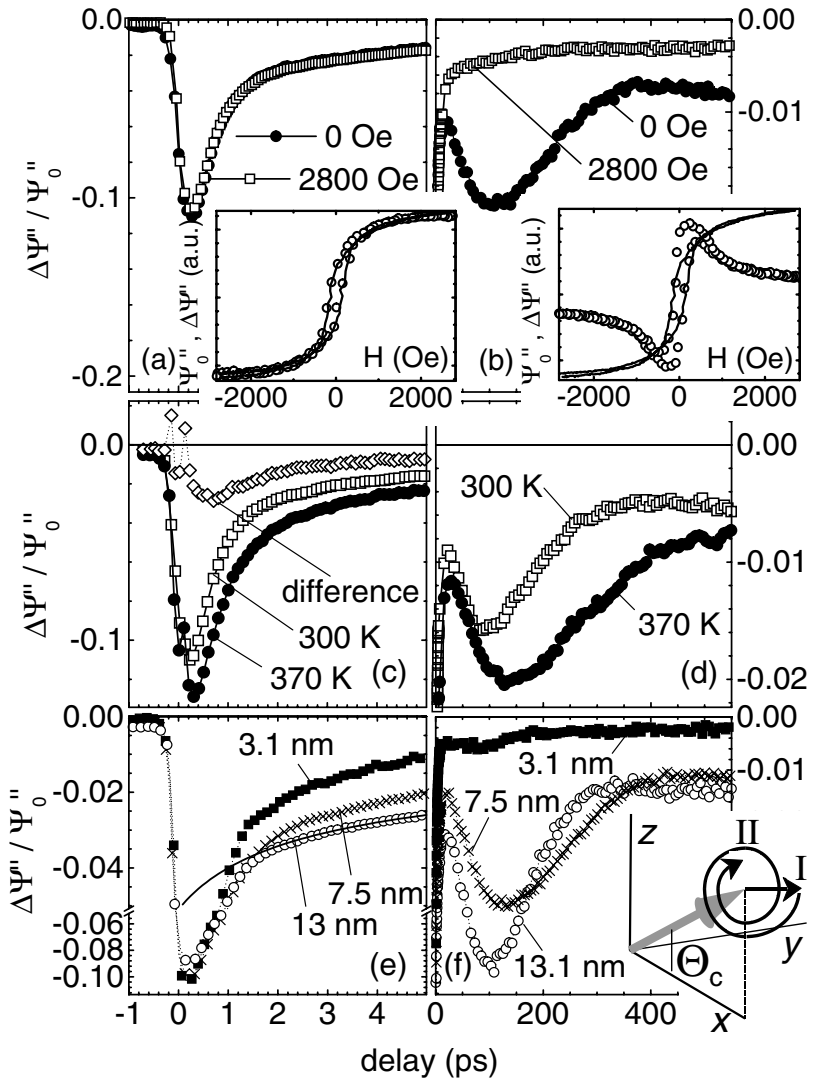

FIG. 2. TRMOKE ellipticity of a $\mathrm{Cu}(111) / \mathrm{Ni} / \mathrm{Cu}$ wedge at a pulse energy $E_{p}=1.6 \mathrm{~nJ}$. Left and right panels display the short ( $<5 \mathrm{ps})$ and long (0-600 ps) term dynamics. (a),(b): Dependence on the applied field (perpendicular to the film) at $4.6 \mathrm{~nm}$ and $300 \mathrm{~K}$. The insets depict $\psi_{0}^{\prime \prime}$ (line) and $\Delta \psi^{\prime \prime}$ (circles) at $0.3 \mathrm{ps}$ (a) and $100 \mathrm{ps}$ (b). (c),(d): Temperature dependence at $4.6 \mathrm{~nm}$ and no applied field, as well as the difference of scans at two different temperatures. (e),(f): Ni thickness dependence at $300 \mathrm{~K}$ and $2800 \mathrm{Oe}$ (e) and $0 \mathrm{Oe}(\mathrm{f})$. The inset in (f), which explains the optically excited precession of $\vec{M}$ after a sudden heating, and the fitted solid line in (e) are discussed in the text. 


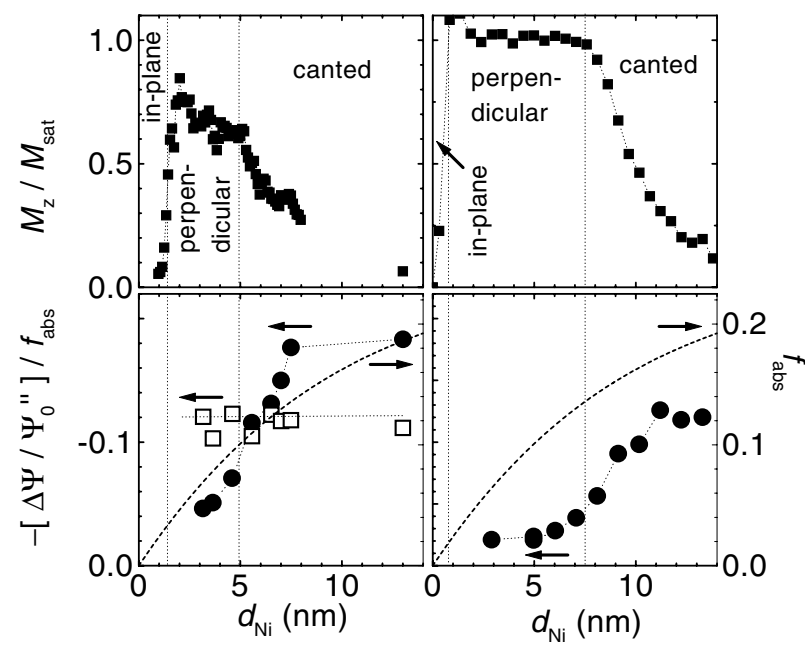

FIG. 3. Static and TRMOKE data as functions of Ni thickness for (001) (left) and (111) (right) oriented wedges. Top: Polar static MOKE remanence. Bottom: Absorption in the Ni film ( $f_{\text {abs }}$, dashed line), and the normalized induced ellipticity (scaled by $\left.f_{\mathrm{abs}}\right)$ at 5 ps (squares) and 100 ps (circles).

that the response scales with the total deposited energy rather than with the initial temperature just after excitation (which is expected to be independent of $d_{\mathrm{Ni}}$ ) suggests that within a couple of picoseconds the excess energy rapidly diffuses out of the Ni film. In general the decay of $\Delta \psi^{\prime \prime}$ can be understood by thermal diffusion. Moreover, for films of $d_{\mathrm{Ni}}>7 \mathrm{~nm}$ and $1.5<t<20 \mathrm{ps}$, a good fit is obtained using the simple expression $\Delta \psi^{\prime \prime} \propto\left(t+t_{0}\right)^{-1 / 2}$ (where $t_{0}$ depends on the diffusion constant $D$ and film thickness). The latter relation is the exact solution for a Gaussian heat profile, and assuming an homogeneous $D$. Figure 2(e) contains a corresponding fit of $\Delta \psi^{\prime \prime} / \psi_{0}^{\prime \prime}(t)$ for the $13 \mathrm{~nm}$ film. The fact that $\Delta \psi^{\prime \prime}$ is described by a simple diffusion model down to $\sim 1.5 \mathrm{ps}$ indicates that at those time scales magnetic relaxation does not play a significant role. We conclude that the demagnetization has been completed already within $1.5 \mathrm{ps}$.

The $\left(t+t_{0}\right)^{-1 / 2}$ dependence of $\Delta \psi^{\prime \prime}$ allows a relatively safe extrapolation to $t=0$. The resulting value, $\Delta \psi^{\prime \prime} / \psi_{0}^{\prime \prime}=-0.048$, corresponds to the induced MO response in equilibrium (i.e., after completion of the demagnetization) if diffusion were negligible. From $f_{\mathrm{abs}}=0.19$ (Fig. 3), a pulse energy $E_{p}=1.6 \mathrm{~nJ}, \quad r=5 \mu \mathrm{m}$, and $C_{p}=28 \mathrm{~J} /(\mathrm{K} \mathrm{mol})$, we estimate $\Delta T \approx 75 \mathrm{~K}$. Substitution of this value and the equilibrium $M(T)$ $\left\{[d M(T) / d T] / M \approx(5.0 \pm 0.5) \times 10^{-4} \mathrm{~K}^{-1}\right.$ at $300 \mathrm{~K}$ [7,8] $\}$ in Eq. (1) yields $\Delta M / M_{0} \approx-0.04$. Its good agreement with the experimentally observed -0.048 confirms that after the first ps the magnetization is dictated by the temperature of the irradiated Ni film. We emphasize that these observations are independent of the crystalline orientation and the applied field. As an example, applying a field perpendicularly to the film $(H \| \hat{z})$ in the canted regime pulls $\vec{M}$ out of plane. As a consequence, both $\psi_{0}^{\prime \prime}$ and $\Delta \psi^{\prime \prime}$ (proportional to $M_{z}$ and $\Delta M_{z}$, respectively) are significantly increased at high $H$ [see the hysteresis loop in Fig. 2(a)]. Nevertheless, the properly normalized $\Delta \psi^{\prime \prime} / \psi_{0}^{\prime \prime}$ is completely unaffected by $\vec{H}$, as demonstrated in Fig. 2(a).

Next we turn our attention to the dynamics during the first hundreds of $f s$. No $H$ dependence, and only a relatively weak $T$ and $d_{\mathrm{Ni}}$ dependence, is observed [Figs. 2(a), 2(c), and 2(e)]. This behavior is consistent with an effect that solely scales with the effective excitation density $f_{\text {exc }}$. The number of excited electrons per $\mathrm{Ni}$ atom follows from $f_{\text {exc }} \approx f_{\text {abs }}\left(d_{\mathrm{Ni}}\right)\left[E_{p} / \hbar \omega\right] V_{\mathrm{Ni}} /\left[\pi r^{2} d_{\mathrm{Ni}}\right] \approx 0.012$, where $V_{\mathrm{Ni}}$ is the $\mathrm{Ni}$ atomic volume. We will argue below that even such a relatively low excitation density suffices for a dichroic bleaching of $\sim 0.1$, being the value of $\Delta \psi^{\prime \prime} / \psi_{0}^{\prime \prime}$ right after excitation in Fig. 2 [9]. It should be realized that out of all states throughout the Brillouin zone (with a total volume $V_{\mathrm{BZ}}$ ) only a selected set (contained in a volume $\left.V_{\text {exc }} \ll V_{\mathrm{BZ}}\right)$ will contribute to the optical response at the given photon energy. Thus, immediately after excitation an enhancement is expected, described by bleaching effects of the order of $f_{\mathrm{exc}} V_{\mathrm{BZ}} / V_{\mathrm{exc}} \gg f_{\mathrm{exc}}$. This indicates that state filling effects may well account for the initial response in the TRMOKE experiments, even if some thermalization takes place within the duration of the laser pulse. A quantative interpretation of the dynamic evolution of the bleaching requires all details of the band structures, matrix elements, etc., which ranges far beyond the scope of this paper.

Obviously, these nonmagnetic contributions hinder a proper assessment of the relaxation time $\tau_{M}$ involved with the ultrafast demagnetization. Nevertheless, a possible separation method is suggested in Fig. 2(c). The difference between two $\Delta \psi^{\prime \prime}$ scans recorded at different $T$ does not show an instantaneous increase at $t=0$, and its first extremum is found around $\sim 0.7 \mathrm{ps}$. Assuming a negligible $T$ dependence of the "state filling" effects, this value might be considered as a rough estimate of $\tau_{M}$.

We continue with discussing the slower processes, which surprisingly appeared after 30 ps. We identified the oscillations as a precession of $\vec{M}$. The possibility to optically launch such a spin precession can be attributed to a thermal modification of the equilibrium spin canting angle $\left(\theta_{c}\right)$. The latter is $T$ dependent through its relation with the uniaxial and cubic anisotropy, described by the $K_{2}$ and $K_{4}$ anisotropy constants, respectively. The mechanism is somewhat resembling to the precession induced by optically modifying the "exchange biasing" between $\mathrm{NiO} / \mathrm{Ni}_{0.8} \mathrm{Fe}_{0.2}$ [10]. An intuitive illustration of the process is found by solving the Landau-LifshitzGilbert (LLG) equation in the limit of weak damping $(\alpha \ll 1)$, and a strong but short heating, as illustrated in the inset of Fig. 2. Within $\tau_{M}$ after arrival of the heating pulse the canting angle $\theta_{c}$ is significantly reduced, which triggers a precession around the perturbed equilibrium direction (phase $\mathrm{I}$ ). After thermal relaxation, $\theta_{c}$ is 
recovered. Nevertheless, due to its initial displacement, $\vec{M}$ starts to precess in its original anisotropy field (phase II). Note that the model predicts an initially negative $\Delta \psi^{\prime \prime} / \psi^{\prime \prime}$, in agreement with experimental observations. Furthermore, our explicit LLG simulations (including the proper damping) revealed the possibility to fit the experimentally observed canting angle, coercive field ( $\sim 200 \mathrm{Oe})$, and resonance frequency $\left(2 \pi / \omega_{\text {res }} \approx 80 \mathrm{ps}\right)$ with a single set (and realistic values) of $K_{2}$ and $K_{4}$.

The field and temperature dependence of the induced oscillation yields a final confirmation of the underlying mechanism. Pulling $\vec{M}$ out of plane in a strong perpendicular field hinders the precession, which is observed as an almost complete quenching of the oscillation [Fig. 2(b)]. Furthermore, the thickness range where the precessive phenomenon manifests itself matches well with the "canted" regime, as shown in Fig. 3. As to the temperature dependence, the decreasing resonance frequency upon heating, shown in Fig. 2(d), is well explained by a thermal softening of the effective magnetic potential.

Finally, we discuss our results in view of some recent publications. We rigorously vitiated the proclaimed evidence reported for an instantaneous demagnetization. Nevertheless, we $d o$ find an ultrafast magnetic response on a ps time scale. The latter statement is based on the agreement of the observed dynamics and $T$ dependence of $\Delta \psi^{\prime \prime} / \psi^{\prime \prime}$ with those expected for thermally induced demagnetization and solving a heat diffusion model. Most probably, the spin equilibration in nickel takes several hundreds of fs up to a ps, as concluded from the thermal difference of $\Delta \psi^{\prime \prime} / \psi^{\prime \prime}$ (diamonds) in Fig. 2(c). This leaves open the possibility of a ferromagnetic to paramagnetic phase transition within $0.5 \mathrm{ps}$, as convincingly demonstrated for $\mathrm{CoPt}_{3}$ [11]. We emphasize that the spin-dependent lifetime (10-20 fs) found in two-photon photoemission [12] is not related to demagnetization, since the electron scattering involved can conserve spin. Also the MO time scales down to the $10 \mathrm{fs}$ in Ni as calculated by Hübner and Zhang [13] are not related to $\tau_{M}$, since the applied Hamiltonian conserves $J_{e}$ explicitly (if neglecting the photons). As to the interpretation of relatively slow ( $>50 \mathrm{ps})$ processes, our experiments have shown that care must be taken to distinguish demagnetization and orientational phenomena. As an example, the $>100$ ps features attributed to a slow "demagnetization" in photoemission studies on Ni [5] might have to be carefully readdressed.

Our new interpretation pushes $\tau_{M}$ from the tens of femtosecond regime towards $\tau_{M} \approx \tau_{\mathrm{ep}}$. This would mean that the lattice degree of freedom may act as a source of angular momentum, thus allowing a nonconservation of $J_{e}$. Still, an extremely efficient spin-lattice relaxation would be required, which might be provided by the high density of states in the Ni $d$ bands, an efficient interface scattering mechanism, or by the presence of a strongly nonequilibrium (hot) phonon system [14]. The latter might distin- guish our case of optical excitation from a perturbation with short magnetic field pulses, in which case a much slower $\tau_{M}=50 \mathrm{ps}$ was found in $\mathrm{Au}$ [15]. In passing, we want to stress that the amount of photons involved in the experiments is by far not enough to contribute a significant $\vec{J}$. In our experiment we typically have $f_{\text {exc }} \approx 0.01$. Combined with the relatively small dichroism in $\mathrm{Ni}(\leq 0.01)$ this yields a direct photon-induced magnetization of at most $10^{-4} \mu_{B}$ per site.

In conclusion, we provided clear evidence for nonmagnetic contributions to the femtosecond MO response in ferromagnetic nickel. An instantaneous demagnetization, as speculated on before, is unlikely. Our rough estimate of the spin relaxation is $0.5-1 \mathrm{ps}$, and may be explained by a highly efficient spin-lattice relaxation. On a slower $(\sim 100 \mathrm{ps})$ time scale we have observed optically induced spin precession. We stress that this effect might not only be of fundamental interest, but could also be applied as all-optical real-time ferromagnetic resonance (FMR). Because of its high spatial resolution as compared to ordinary FMR, we foresee a high application potential in the research on microscopic magnetic elements relevant for data storage and spin electronic devices.

We acknowledge E. Beaurepaire, J. Hohlfeld, R. Vollmer, W. Hübner, and P.J. van Hall for enlightening scientific discussions. This work forms part of the research programme of the Dutch foundation for the Fundamental Research on Matter (FOM).

*Email address: koopmans@phys.tue.nl

[1] E. Beaurepaire et al., Phys. Rev. Lett. 76, 4250 (1996).

[2] J. Hohlfeld et al., Phys. Rev. Lett. 78, 4861 (1997).

[3] J. Güdde et al., Phys. Rev. B 59, R6608 (1999).

[4] B. Koopmans et al., J. Appl. Phys. 87, 5070 (2000).

[5] A. Scholl et al., Phys. Rev. Lett. 79, 5146 (1997).

[6] R. Jungblut et al., J. Appl. Phys. 75, 6424 (1994).

[7] P. Weiss and R. Forrer, Ann. Phys. (Leipzig) 5, 153 (1926).

[8] Numerical Data and Functional Relationships in Science and Technology, edited by K.-H. Hellwege and O. Madelung, Landolt-Börnstein, New Series, Group III, Vol. 19a (Springer-Verlag, Berlin, 1986).

[9] For a $\mathrm{Ni}$ thin film on a $\mathrm{Cu}$ substrate the ellipticity $\left(\psi^{\prime \prime}\right)$ corresponds approximately to the imaginary (i.e., absorptive) part of the dichroism. Thus, one should compare here to values of $\Delta \psi^{\prime \prime} / \psi_{0}^{\prime \prime}$. The rotation $(\Psi /)$ corresponds roughly to the real (refractive) part, which may display a more nontrivial response, such as the bipolar oscillation observed around zero time delay in Fig. 1.

[10] G. P. Ju et al., Phys. Rev. Lett. 82, 3705 (1999).

[11] E. Beaurepaire et al., Phys. Rev. B 58, 12134 (1998).

[12] M. Aeschlimann et al., Phys. Rev. Lett. 79, 5158 (1997).

[13] W. Hübner and G.P. Zhang, Phys. Rev. B 58, R5920 (1998).

[14] P. J. van Hall (to be published).

[15] A. Y. Elezzabi, M. R. Freeman, and M. Johnson, Phys. Rev. Lett. 77, 3220 (1996). 